\title{
A Hemorrhagic Cholecystitis in a Penetrating Hepatic Injury
}

\author{
Kang Kook Choi, Min A Lee, Dae Sung Ma, Gil Jae Lee, Byung chul Yu, Jung Nam Lee \\ Department of Trauma Surgery, Gachon University, Gil Medical Center, Incheon, Korea
}

A penetrating hepatic trauma can be accompanied by gallbladder (GB) injury. It is often detected by abdominal computed tomography (CT) but missed without CT. One should consider the possibility of GB injury through the hepatic side in patients with penetrating hepatic trauma adjacent to the GB.

Key Words: Cholecystitis, Acute; Hemorrhage; Liver; Lung; Injuries

(Trauma Image Proced 2017(1):15-16)

\section{CASE}

A 65-year-old man was transferred to the trauma bay. He was stabbed in the fifth intercostal space of the right chest and right upper quadrant of the abdomen. The patient was drowsy on arrival to the trauma center. His initial systolic blood pressure was $90 \mathrm{mmHg}$, heart rate was 132 beats/min, respiratory rate was 22 breaths/min, and body temperature was $35.8^{\circ} \mathrm{C}$. Exploratory thoracotomy and laparotomy revealed penetration of the right lung. The diaphragm had a 5-cm long penetrating wound, and two penetrating wounds were identified in

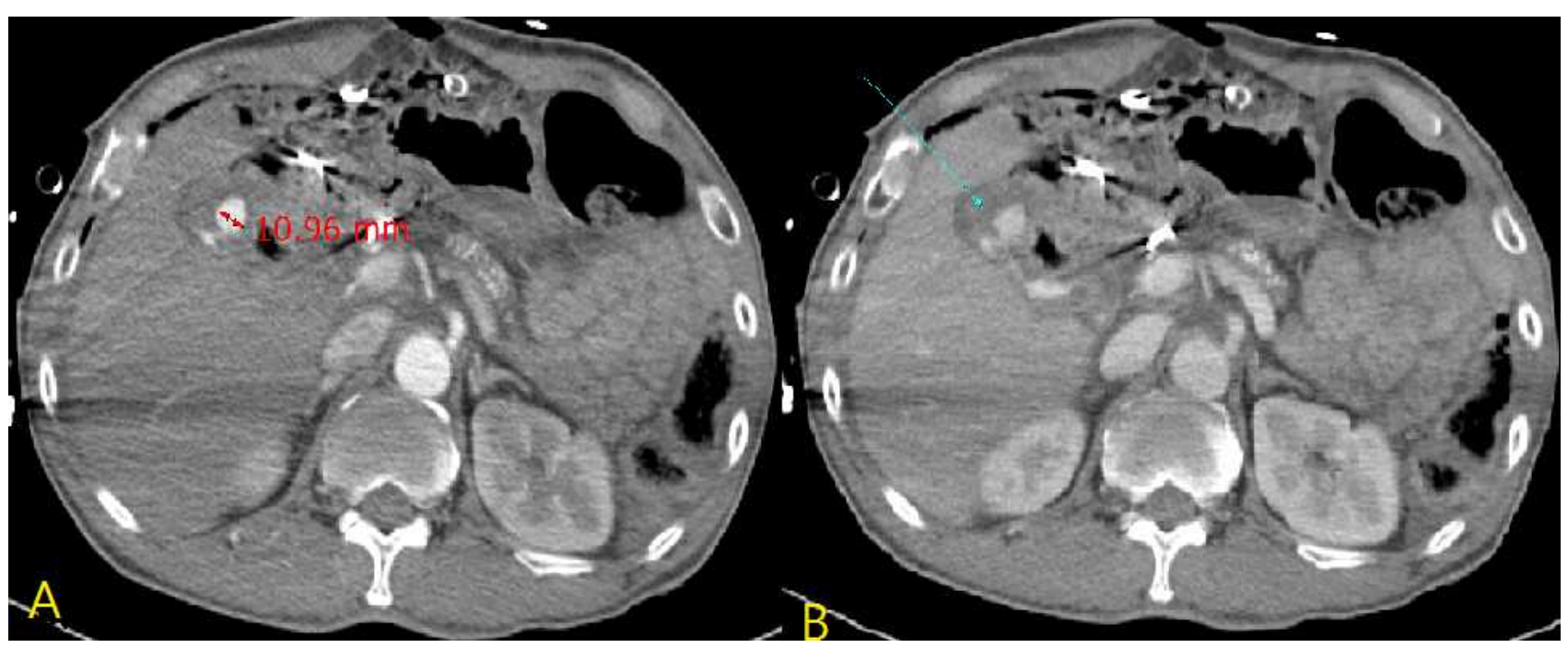

Fig. On postoperative day 1, abdominal CT reveals contrast extravasation in GB and GB wall thickening (A:arterial phase, B:venous phase)

Received: March 5, 2017 Revised: April 12, 2017 Accepted: May 1, 2017

Correspondence to: Jung Nam Lee, Department of Trauma Surgery, Gachon University, Gil Medical Center, 21, 774 beon-gil, Namdongdaero, Namdong-gu, Incheon 21565, Korea

Tel: 82-51-240-7369, Fax: 82-51-240-7719, E-mail: calvin511@naver.com

Copyright (c) 2017 Korean Association for Research, Procedures and Education on Trauma. All rights reserved.

@This is an open-access article distributed under the terms of the Creative Commons Attribution Non-Commercial License (http://creativecommons.org/ licenses/by-nc/4.0) which permits unrestricted noncommercial use, distribution, and reproduction in any medium, provided the original work is properly cited 
hepatic segments 4 and 8 . The penetrating hepatic wounds were sutured. For the injured lung, wedge resection was performed using a stapler. After perihepatic packing, angioembolization was performed for the penetrating hepatic wound because the wounds were deep and the operator was not convinced that hemostasis was successful. Angioembolization revealed active arterial bleedings from hepatic segment 8 and the cystic artery and were successfully embolized. On postoperative day 1 , abdominal $\mathrm{CT}$ revealed contrast extravasation in GB (Fig.). The vital signs were stable at that time. On postoperative day 2 , a second-look operation revealed that GB was necrotized and distended with hematoma. The packing gauzes were removed, and cholecystectomy was performed. The patient was discharged without any complications 15 days after the initial operation.

\section{DISCUSSION}

Acute cholecystitis can appear in trauma patients whether the trauma is blunt or penetrating (1). In penetrating hepatic trauma, the possibility of hidden GB injury always exists. If GB injury is suggested, abdominal CT or ultrasonography can be a good diagnostic option and a cholecystectomy is the simple and best treatment of choice.

\section{REFERENCES}

1. Parekh J, Corvera CU. Hemorrhagic cholecystitis. Archives of surgery. 2010;145(2):202-4. 